\title{
Magnetic-Field-Induced insulator-conductor transition in quenched lattice gauge theory
}

\author{
P. V. Buividovich*广 \\ ITEP, 117218 Russia, Moscow, B. Cheremushkinskaya str. 25 \\ JINR, 141980 Russia, Moscow region, Dubna, Joliot-Curie str. 6 \\ E-mail: buividovicheitep.ru
}

\section{N. Chernodub}

Laboratoire de Mathematiques et Physique Theorique, CNRS UMR 6083, Fédération Denis Poisson, Université de Tours, Parc de Grandmont, F37200, Tours, France

DMPA, University of Gent, Krijgslaan 281, S9, B-9000 Gent, Belgium

ITEP, 117218 Russia, Moscow, B. Cheremushkinskaya str. 25

\section{T. Kalaydzhyan}

DESY Hamburg, Theory Group, Notkestrasse 85, D22607 Hamburg, Germany

ITEP, 117218 Russia, Moscow, B. Cheremushkinskaya str. 25

\section{E. Kharzeev}

Physics Department, Brookhaven National Laboratory, Upton, NY 11973-5000, USA

Department of Physics and Astronomy, Stony Brook University, Stony Brook, NY 11794-3800, USA

\section{E. V. Luschevskaya}

ITEP, 117218 Russia, Moscow, B. Cheremushkinskaya str. 25

\section{I. Polikarpov}

ITEP, 117218 Russia, Moscow, B. Cheremushkinskaya str. 25

We study the electric conductivity of a quenched $S U$ (2) lattice gauge theory in both confinement and deconfinement phases in the presence of constant external electromagnetic field. The conductivity is extracted from the current-current correlation functions using the Kubo formulas and the Maximal Entropy Method. We find that at small but nonzero temperatures in the confinement phase the external magnetic field induces nonzero electric conductivity along the direction of the magnetic field, transforming the system from an insulator into an anisotropic conductor. In the deconfinement phase the conductivity does not exhibit any sizable dependence on the magnetic field.

The XXVIII International Symposium on Lattice Filed Theory

June 14-19,2010

Villasimius, Sardinia Italy

\footnotetext{
* Speaker.

$\dagger$ The author was supported by personal grants from the "Dynasty" foundation and from the FAIR-Russia Research Center and by the RFBR grant 09-02-00338-a
} 


\section{Introduction}

The so-called Chiral Magnetic Effect (CME) [1] in hadronic matter has attracted recently a lot of attention. This is the name for the phenomenon of the generation of electric current along the direction of the external magnetic field in the background of topologically nontrivial gauge field configurations. Experimentally, the effect manifests itself as the dynamical enhancement of fluctuations in the numbers of charged hadrons emitted above and below the reaction plane in off-central heavy-ion collisions. The STAR collaboration at RHIC claimed the experimental observation of this effect.

The signatures of the CME have been also studied on the lattice, and the evidence for enhanced charge fluctuations in magnetic field has been found [2, 3]. In [2] it was found that the fluctuations of the electric current along the magnetic field are strongly enhanced as compared to the fluctuations of current in the perpendicular directions. This conclusion was also confirmed by an analytical calculadtion in the instanton gas model [4].

As is well known, the fluctuational spectra of the electromagnetic current can be related to the conductivity of the media via the fluctuation-dissipation theorem. The low-frequency part of the spectrum corresponds to the standard ohmic DC conductivity [5]. In this paper we calculate the tensor of electric conductivity in $S U$ (2) lattice gauge theory with quenched chirally invariant quarks [6] and study its volume dependence. We find that in the magnetic field the vacuum state of this theory acquires electric conductivity in the direction of the field, thus becoming an anisotropic conductor [7].

Electric conductivity can be calculated from the correlator of vector currents $j_{i}(x)=\bar{q}(x) \gamma_{i} q(x)$ :

$$
G_{i j}(\tau)=\int d^{3} \vec{x}\left\langle j_{i}(\overrightarrow{0}, 0) j_{j}(\vec{x}, \tau)\right\rangle
$$

According to the fluctuation-dissipation theorem, let us define the spectral function $\rho(w)$ which corresponds to the correlator (1.1) [8]:

$$
\begin{aligned}
G_{i j}(\tau) & =\int_{0}^{+\infty} \frac{d w}{2 \pi} K(w, \tau) \rho_{i j}(w), \\
K(w, \tau) & =\frac{w}{2 T} \frac{\cosh \left(w\left(\tau-\frac{1}{2 T}\right)\right)}{\sinh \left(\frac{w}{2 T}\right)},
\end{aligned}
$$

where $T$ is the temperature. This definition differs from the standard one with $K(w, \tau)=\frac{\cosh \left(w\left(\tau-\frac{1}{2 T}\right)\right)}{\sinh \left(\frac{w}{2 T}\right)}$, but has an advantage that the value of the conductivity is related to the value of the spectral density $\rho_{i j}(w \rightarrow 0)$ rather than to its slope and is thus numerically more stable [8]. The Kubo formula for the electric conductivity then reads $[5,8]$ :

$$
\sigma_{i j}=\lim _{\omega \rightarrow 0} \frac{\rho_{i j}(\omega)}{4 T} .
$$

The correlator (1.1) is measured on an equilibrium ensemble of $S U(2)$ gauge fields. Since the origin of charge fluctuations in CME is associated with chirality fluctuations [1], we use the overlap lattice Dirac operator $\mathscr{D}$ with exact chiral symmetry [6] to measure the vector currents. We 
consider the two-current correlator in the meson channel, which is represented in terms of Dirac propagators in a fixed Abelian and non-Abelian gauge fields and is then averaged over non-Abelian gauge fields:

$$
\begin{array}{r}
\left\langle\bar{q}(x) \gamma_{i} q(x) \bar{q}(y) \gamma_{j} q(y)\right\rangle= \\
=\int \mathscr{D} A_{\mu} e^{-S_{Y M}\left[A_{\mu}\right]} \operatorname{Tr}\left(\frac{1}{\mathscr{D}+m} \gamma_{i} \frac{1}{\mathscr{D}+m} \gamma_{j}\right),
\end{array}
$$

where $S_{Y M}\left[A_{\mu}\right]$ is the lattice action for non-Abelian gauge fields $A_{\mu}$. Uniform magnetic field is added to the Dirac operator by substituting $s u(2)$-valued vector potential $A_{\mu}$ with $u(2)$-valued one $A_{\mu i j} \rightarrow A_{\mu i j}+1 / 2 F_{\mu \nu} x_{\nu} \delta_{i j}$. In order to account for periodic boundary conditions we introduce an additional boundary twist for fermions $[2,9]$. We use the tadpole-improved Wilson-Symanzik action on the lattice (see, e.g., the expression (1) in [10]). To obtain the Dirac propagator, we implement the Shifted Unitary Minimal Residue method (SHUMR) described in [11]. The parameters of equilibrium gauge field ensembles which we use are summarized in Table 1. Uniform magnetic field is introduced into the Dirac operator as described in [2].

\begin{tabular}{|c|c|c|c|c|}
\hline$\beta$ & $\mathrm{a}, \mathrm{fm}$ & $N_{s}^{3} \times N_{t}$ & $T / T_{c}$ & \#conf \\
\hline 3.2810 & 0.102 & $14^{3} \times 14$ & 0.43 & 30 \\
3.2810 & 0.102 & $16^{3} \times 16$ & 0.38 & 30 \\
3.3555 & 0.089 & $16^{3} \times 16$ & 0.43 & 30 \\
3.3250 & 0.095 & $16^{3} \times 6$ & 1.12 & 30 \\
\hline
\end{tabular}

Table 1: Lattice parameters used in our simulations. The critical temperature of the confinementdeconfinement phase transition in quenched $S U$ (2) lattice gauge theory is $T_{c}=313$.(3) $\mathrm{MeV}$ [12].

The current-current correlators at different temperatures and magnetic fields are plotted on Fig. 1. The data are for the $14^{4}$ lattice with spacing $a=0.102 \mathrm{fm}$ (left) and for the $16^{3} \times 6$ lattice with spacing $a=0.095 \mathrm{fm}$ (right). For the latter lattice the temperature is $T=350 \mathrm{MeV}>T_{c}$ and the theory is in the deconfinement phase.

Without the magnetic field the correlators have a typical exponential decay in the confinement phase. In the deconfinement phase the decay is significantly slower for all $G_{i i}(\tau)$. In a magnetic field with the strength $q B=(0.63 \mathrm{GeV})^{2}$, in the confinement phase the correlator $G_{z z}(\tau)$ decays much slower and is significantly larger than zero for all $\tau$, much like in the deconfinement phase. On the other hand, for the perpendicular components of the current $G_{x x}(\tau)$ and $G_{y y}(\tau)$ the correlators decay somewhat quicker than in the zero field case. In the deconfinement phase all the correlators are practically unaffected by the magnetic field.

In order to extract the spectral functions (1.2) from the correlators (1.1), we apply the Maximal Entropy Method $[8,13,14]$. Our analysis is similar to that of $[8,14]$, with the default model $\bar{m}(w)=\bar{m}_{0}(b+a w)$ [8]. Some spectral functions at different temperatures and magnetic fields are shown on Fig. 1.

In the absence of magnetic field below the deconfinement phase transition, the spectral function has a distinct peak near $w \approx 1 \mathrm{GeV}$, which corresponds to the mass of the $\rho$ meson in quenched $S U$ (2) lattice gauge theory $[14,15]$. The width of this peak in quenched approximation is a lattice artefact [14], and should decrease for finer and larger lattices. The spectral function in the limit 

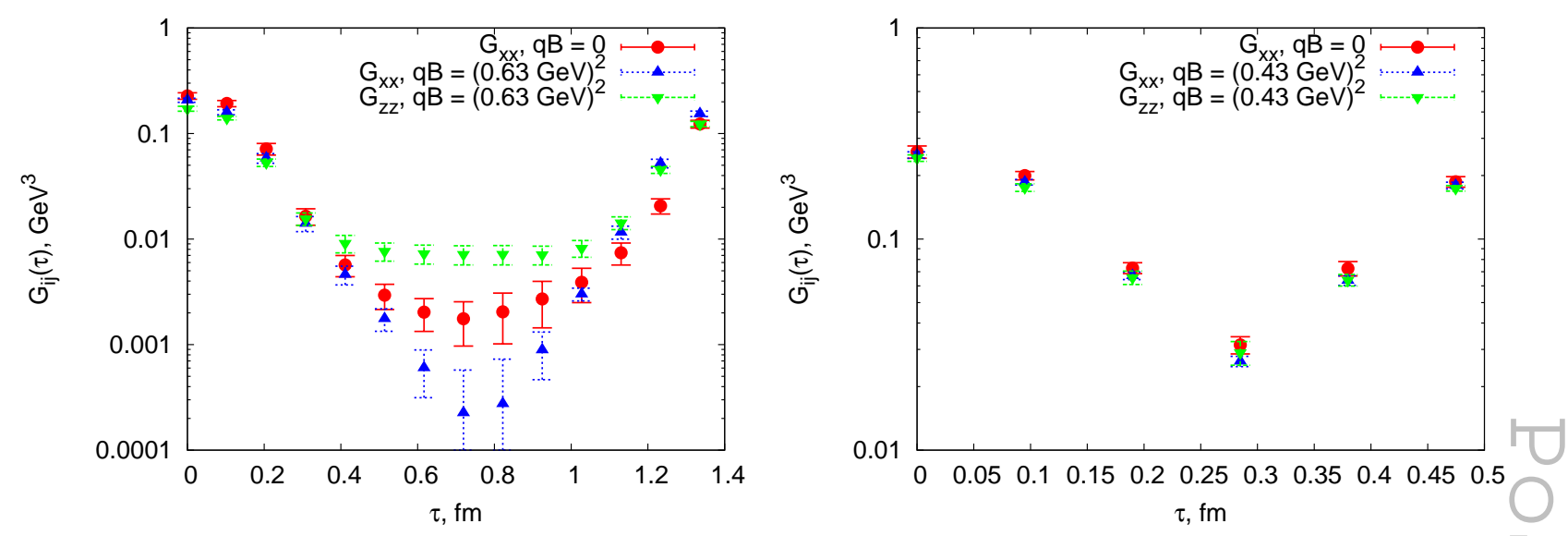

Figure 1: The correlator (1.1) in the confinement (left) and in the deconfinement phases (right) at $T=$ $350 \mathrm{MeV}$.
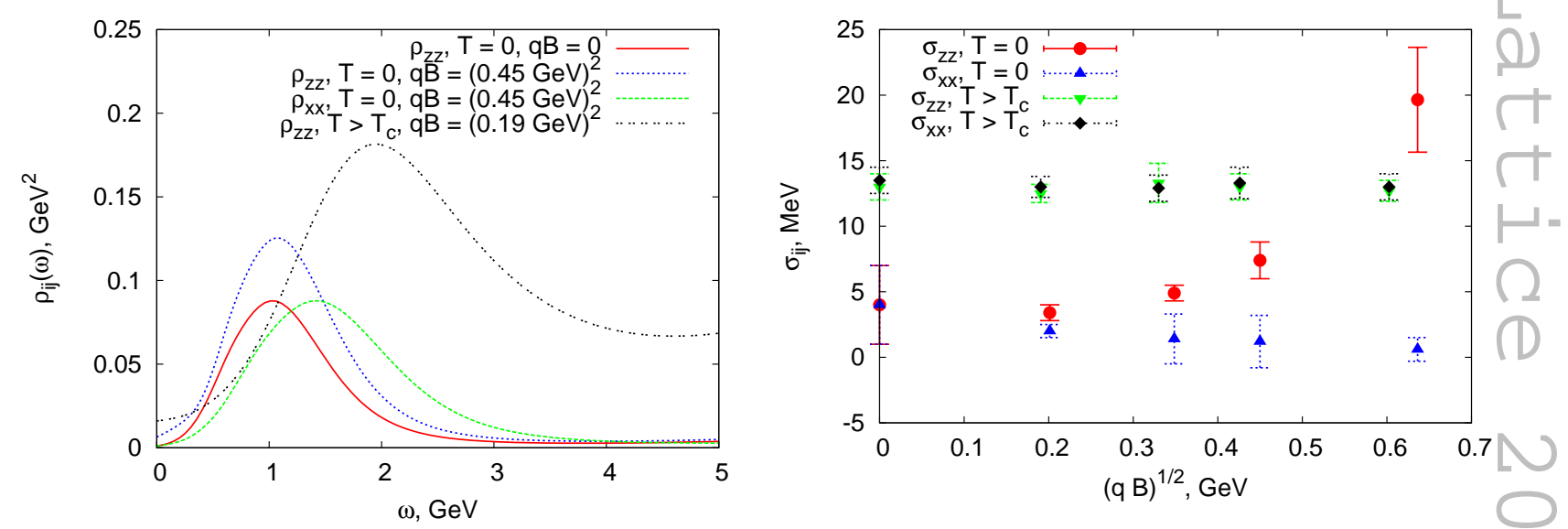

Figure 2: On the left: Spectral functions $\rho_{i j}(w)$ in the confinement and deconfinement phases. On the right: electric conductivity as a function of external magnetic field in the confinement and deconfinement phases. The points for $\sigma_{z z}$ and $\sigma_{x x}$ at $T>T_{c}$ coincide within error range.

of zero frequency, $\rho_{i j}(0)$, is compatible with zero. Correspondingly, in the absence of external magnetic field the vacuum of quenched QCD does not conduct DC current, in agreement with the previous lattice results $[7,13,14]$. When the external magnetic field is applied, the peak becomes more pronounced and the spectral density at small frequencies becomes nonzero. For other components of $\rho_{i j}(w)$ nothing changes qualitatively, but the peak which corresponds to the $\rho$-meson becomes somewhat smaller and shifts slightly to larger $w$. The conductivity remains equal to zero within error range. Thus when the external magnetic field is applied to quenched vacuum of $S U$ (2) lattice gauge theory, it acquires nonzero conductivity, but only in the direction of the magnetic field.

In the deconfinement phase, the spectral function is nonzero at $w=0$ already at zero magnetic field and has a smooth peak near $w \approx 2 \mathrm{GeV}$. Thus the deconfined phase of the quenched $S U(2)$ lattice gauge theory is a conductor $[7,8,13]$. Since the shape of the correlator $G_{i j}(\tau)$ is practically unaffected by the magnetic field, the spectral function $\rho_{i j}(w)$ and the conductivity $\sigma_{i j}$ are also independent of the magnetic field. 
The dependence of the electric conductivity $\sigma_{i j}$ on the external magnetic field is illustrated on Fig. 2 both below and above the deconfinement phase transition. In the deconfinement phase the temperature is $T=350 \mathrm{MeV}$. The value of the conductivity was extracted from the value of the spectral function at $w=0$ using (1.4). In the confinement phase and at zero magnetic field the conductivity is zero within error range. As the magnetic field is turned on, the conductivity $\sigma_{z z}$ in the direction of the magnetic field grows, while all other components of $\sigma_{i j}$ remain equal to zero within error range. In the deconfinement phase the conductivity is isotropic and is practically independent of the magnetic field. One can not exclude, of course, that there is a weak anisotropy, which cannot be seen at the small number of configurations that we have. It should be also noted that in our simulations the value of conductivity $\sigma=15 \pm 2 \mathrm{MeV}$ at $T=350 \mathrm{MeV}>T_{c}$ is still much smaller than the results obtained in $[8,13]$ in quenched $S U(3)$ lattice gauge theory with light staggered fermions. This difference is likely to be an artefact of a quenched theory, since in this case different probes of the confinement-deconfinement phase transition might give different transition temperatures. In particular, while in quenched $S U(2)$ lattice gauge theory the Polyakov loop goes to zero at $T_{c}=313$. (3) $\mathrm{MeV}$ [12], the chiral condensate is not zero above this temperature [16]. The situation might be similar for the insulator-conductor transition, which in the quenched case might be replaced by a soft crossover with much smaller conductivity at $T>T_{c}$.

The transport coefficients typically have rather strong dependence on lattice parameters. To ensure that the nonzero conductivity is not a finite-volume artefact, we have also performed the simulations at different lattice volumes and lattice spacings (see Table 1). The values of conductivity $\sigma_{z z}$ for different lattice parameters are plotted on Fig. 3. One can see that as we go to finer and larger lattices, the conductivity does not change within statistical errors.
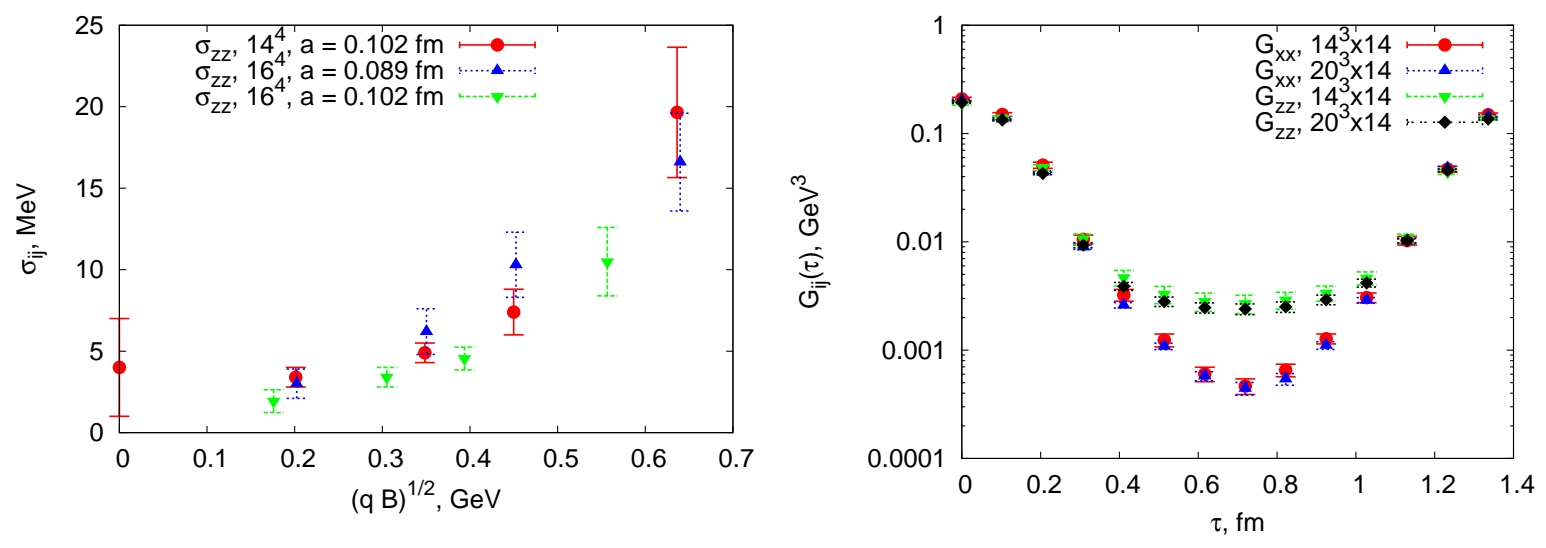

Figure 3: On the left: electric conductivity in the direction of external magnetic field $\sigma_{z z}$ for different lattice parameters. On the right: a comparison of current-current correlators (1.1) calculated at $14^{3} \times 14$ and $20^{3} \times 14$ lattices at equal values of the lattice spacing and magnetic field strength $\sqrt{q B}=0.45 \mathrm{GeV}$.

Strictly speaking, the expressions (1.1) and (1.4) are only valid at finite temperature, while we work with the $14^{4}$ lattices which are symmetric in all space-time directions and hence correspond to zero temperature in the standard lattice lore. However, one can still apply the expressions (1.1), (1.4) with some small but finite temperature $T=\left(N_{t} a\right)^{-1}$, since real lattices have finite extent $N_{t}$ in time direction. In this case one should make sure that the effects of the finite spatial volume in the nonlocal observables such as the current-current correlator are insignificant. To this end, 
we plot the correlators (1.1) calculated on the $14^{3} \times 14$ and $20^{3} \times 14$ lattices at magnetic field strength $\sqrt{q B}=0.45 \mathrm{GeV}$ on Fig. 3. The correlators calculated on different lattices indeed agree within error bars for the currents both parallel and perpendicular to the magnetic field. Thus for the correlators under consideration finite-volume effects seem to be negligible. Correspondingly, the results presented here and in [7] refer to the case of small but finite temperature (below the deconfinement phase transition) rather than to exactly zero temperature.

Thus a strong magnetic field can turn a vacuum state of non-Abelian gauge theory from insulator into an anisotropic conductor. According to some models, this new state can be even a superconductor above some critical magnetic field [17]. Such transitions are often encountered in condensed-matter physics. The deconfined phase of non-Abelian gauge theory, at least in our quenched $S U$ (2) case, is an isotropic conductor, and the conductivity is practically independent of the magnetic field. Instanton models also suggest that at higher temperatures the instanton contribution to the CME becomes negligible [4].

Finally, let us comment possible experimental indications of anisotropic conductivity of hadronic matter. The v.e.v. $\left\langle j_{k}(x) j_{l}(y)\right\rangle$ is related to the polarization of soft virtual photons and, hence, to the angular correlations of soft dilepton pairs emitted in the collision process [13, 18, 19]. According to the results presented above, the conductivity tensor can be represented as $\sigma_{i j} \sim B_{i} B_{j}$. The dilepton emission rate in the dilepton center of mass frame is $[18,19]$ :

$$
\frac{R}{V} \sim \int \frac{d^{3} p}{E(p)}\left(\vec{B}^{2}-(\vec{B} \cdot \vec{n})^{2}\right) \sim \sin ^{2}(\theta)
$$

where $\vec{n}$ is the unit vector in the direction of the momentum of one of the leptons and $\theta$ is the angle between $\vec{n}$ and the direction of the magnetic field. Therefore, there should be more dileptons in the direction perpendicular to the reaction plane, which can be observed as a negative elliptic flow for soft photons and leptons.

\section{References}

[1] D. E. Kharzeev, L. D. McLerran, H. J. Warringa, The effects of topological charge change in heavy ion collisions: Event by event P and CP violation, Nucl. Phys. A 803 (2008) 227 [ArXiv:0711.0950].

[2] P. V. Buividovich, M. N. Chernodub, E. V. Luschevskaya, M. I. Polikarpov, Numerical evidence of chiral magnetic effect in lattice gauge theory, Phys. Rev. D 80 (2009) 054503 [ArXiv:0907.0494].

[3] M. Abramczyk, T. Blum, G. Petropoulos, R. Zhou, Chiral magnetic effect in 2+1 flavor $Q C D+Q E D$, in proceedings of Lattice 2009, POS (LAT2009) 181.

[4] Seung-Il Nam, Chiral magnetic effect at low temperature, Phys. Rev. D 80 (2009) 114025 [ArXiv:0911.0509].

[5] L. P. Kadanoff P. C. Martin, Hydrodynamic equations and correlation functions, Ann. Phys. 24 (1963) 19. 
[6] H. Neuberger, Exactly massless quarks on the lattice, Phys. Lett. B 417 (1998) 141 [ArXiv:hep-lat/9707022].

[7] P.V. Buividovich, M.N. Chernodub, D.E. Kharzeev, T. Kalaydzhyan, E.V. Luschevskaya, M.I. Polikarpov, Magnetic-field-induced insulator-conductor transition in $S U(2)$ quenched lattice gauge theory, Phys. Rev. Lett. 105 (2010) 132001 [ArXiv: 1003 .2180].

[8] G. Aarts, C. Allton, J. Foley, S. Hands, S. Kim, Spectral functions at small energies and the electrical conductivity in hot, quenched lattice QCD, Phys. Rev. Lett. 99 (2007) 022002 [ArXiv: hep-lat/0703008].

[9] M. H. Al-Hashimi, U.-J. Wiese, Discrete accidental symmetry for a particle in a constant magnetic field on a torus, Annals Phys. 324 (2009) 343 [ArXiv: 0807.0630 ].

[10] V. G. Bornyakov, E. V. Luschevskaya, S. M. Morozov, M. I. Polikarpov, E.-M. Ilgenfritz, M. Müller-Preussker, The topological structure of $S U(2)$ gluodynamics at $T>0$ : an analysis using the Symanzik action and Neuberger overlap fermions, Phys. Rev. D 79 (2009) 054505 [ArXiv:0807.1980].

[11] A. Borici A. Allkoci, Shifted unitary orthogonal methods for the overlap inversion, in proceedings of Lattice 2005, POS (LAT2005) 101.

[12] V. G. Bornyakov, E.-M. Ilgenfritz, B. V. Martemyanov, S. M. Morozov, M. Müller-Preussker, A. I. Veselov, Calorons and dyons at the thermal phase transition analyzed by overlap fermions, Phys. Rev. D 76 (2007) 054505 [ArXiv: 0706.4206 ].

[13] S. Gupta, The electrical conductivity and soft photon emissivity of the QCD plasma, Phys. Lett. B 597 (2004) 57 [ArXiv: hep-lat/ 0301006 ].

[14] M. Asakawa, T. Hatsuda, Y. Nakahara, Maximum entropy analysis of the spectral functions in lattice QCD, Prog. Part. Nucl. Phys. 46 (2001) 459 [ArXiv: hep-lat/ 011040 ].

[15] P. Hasenfratz, S. Hauswirth, K. Holland, T. Jorg, F. Niedermayer, Testing the fixedpoint QCD action and the construction of chiral currents, Nucl. Phys. B 643 (2002) 280 [ArXiv:hep-lat/0205010].

[16] P. V. Buividovich, E. V. Luschevskaya, M. I. Polikarpov, Finite-temperature chiral condensate and low-lying Dirac eigenvalues in quenched SU(2) lattice gauge theory, Phys. Rev. D 78 (2008) 074505 [ArXiv: 0809.3075$].$

[17] M. N. Chernodub, Superconductivity of QCD vacuum in strong magnetic field, Phys. Rev. $D$ 82 (2010) 085011 [ArXiv: 1008.1055$].$

[18] L. D. McLerran T. Toimela, Photon and dilepton emission from the quark-gluon plasma: Some general considerations, Phys. Rev. D 31 (1985) 545.

[19] E. L. Bratkovskaya, O. V. Teryaev, V. D. Toneev, Anisotropy of dilepton emission from nuclear collisions, Phys. Lett. B 348 (1995) 283. 